\title{
Clinical Analysis of Bloodstream Infections During Agranulocytosis After Allogeneic Hematopoietic Stem Cell Transplantation
}

This article was published in the following Dove Press journal: Infection and Drug Resistance

\author{
Weijie Cao ${ }^{1, *}$ \\ Lina Guan ${ }^{1} * *$ \\ Xiaoning $\mathrm{Li}^{\prime}$ \\ Ran Zhang' \\ $\mathrm{Li} \mathrm{Li}{ }^{\prime}$ \\ Suping Zhang' \\ Chong Wang' \\ Xinsheng Xie' \\ Zhongxing Jiang' \\ Dingming Wan' \\ Xiaohui $\mathrm{Chi}^{2}$
}

'Department of Hematology, The First Affiliated Hospital of Zhengzhou University, Zhengzhou, People's Republic of China; ${ }^{2}$ Collaborative Innovation Center for Diagnosis and Treatment of Infectious Diseases, State Key Laboratory for Diagnosis and Treatment of Infectious Diseases, The First Affiliated Hospital, College of Medicine, Zhejiang University, Hangzhou, People's Republic of China

*These authors contributed equally to this work
Purpose: To explore the epidemiological characteristics and risk factors of bloodstream infections (BSI) in patients who develop agranulocytosis fever after allogeneic hematopoietic stem cell transplantation (allo-HSCT). This study also provides a basis for the clinical treatment of BSI.

Methods: A retrospective analysis of 397 allo-HSCT patients in the Department of Hematology of our hospital was conducted from January 2013 to December 2017 to analyze the incidence of BSI, the distribution and types of pathogenic bacteria, and drug resistance rates. We also determined whether various parameters are risk factors to BSI, including the patient age, gender, disease type, transplantation method, stem cell source, pre-treatment with anti-thymocyte globulin (ATG), and agranulocytosis time.

Results: Among the 397 allo-HSCT patients, 294 had a fever during the period of agranulocytosis, and 52 cases were found to have BSI. The incidence of BSI in patients with agranulocytosis fever was $17.7 \%$ (52/294). Among the 60 pathogens detected, 43 (71.67\%), $10(16.67 \%)$, and $7(11.67 \%)$ were Gram negative strains, Gram positive strains, and fungi, respectively. The most common bacteria were Escherichia coli, Klebsiella pneumoniae, and Pseudomonas aeruginosa. The detection rate of extended-spectrum $\beta$-lactamase (ESBL) was $40.0 \%$, and carbapenem-resistant Enterobacteriaceae (CRE) accounted for 17.9\%. Singlefactor and multi-factor analyses showed that pre-treatment with ATG, agranulocytosis time ( $\geq 21$ days), and stem cell source were risk factors for BSI.

Conclusion: We found that in our hospital, BSIs in allo-HSCT patients are mainly caused by Gram-negative bacteria, and the resistance rate to carbapenem drugs is high. Pre-treatment with ATG, agranulocytosis time ( $\geq 21$ days), and stem cell source are risk factors for BSI.

Keywords: hematopoietic stem cell transplantation, agranulocytosis, BSI, pathogenic bacteria, risk factors

\section{Introduction}

Allogeneic hematopoietic stem cell transplantation (allo-HSCT) is a treatment process for restoring normal hematopoietic and immune functions. In this method, patients undergo high-dose radiotherapy and chemotherapy, and immunosuppressive pre-treatment is done to eliminate abnormal hematopoietic and immune systems. The patient is then transfused with allogeneic hematopoietic stem cells. This strategy is an effective cure for blood diseases, bone marrow failure syndrome, and immune deficiency. ${ }^{1,2}$ However, neutrophil deficiency, impaired mucosal barrier, and weakened immune function typically occur after transplantation, which increases the risk of infection after HSCT. $^{3}$ 
Bloodstream infections (BSI) are a severe type of systemic infectious disease caused by the invasion of the circulatory system by pathogenic microorganisms. Notably, BSI is a common complication in the early stages of allo-HSCT and has an incidence rate of $13.6 \%$ $38.9 \%{ }^{4-7}$ According to literature, the occurrence of bloodstream infections is a huge risk factor to early deaths after HSCT. ${ }^{8-10}$ The occurrence of BSI after HSCT is exacerbated by the widespread use of antibiotics and the resultant antibacterial resistance, especially multi-drugresistant bacteria (MDR) that seriously affects the survival of transplant patients. ${ }^{11-13}$ Thus, evaluation of the distribution and prevalence of drug-resistant pathogens of the bloodstream in allo-HSCT patients and the study of the BSI risk factors could guide the course of clinical treatment for BSI prevention and control. This study retrospectively analyzed the BSI risk factors in patients with allo-HSCT in the First Affiliated Hospital of Zhengzhou University from 2013 to 2017 . The detection rate, distribution, and drug sensitivity of pathogenic bacteria after allo-HSCT was also evaluated.

\section{Materials and Methods}

\section{Case Information}

From January 2013 to December 2017, 397 patients who received allogeneic HSCT for the treatment of hematological diseases in the First Affiliated Hospital of Zhengzhou University were selected. The patients included 242 males and 155 females, with a median age of 21 (1-62) years. Of these, 115 cases had acute myeloid leukemia (AML), 110 with severe aplastic anemia (SAA), 102 with acute lymphocytic leukemia (ALL), and 70 patients with other conditions.

\section{Type of Transplantation}

According to the difference in the histocompatible typing and relationship, allo-HSCT is divided into matched sibling transplantation, partially matched related transplantation and matched unrelated transplantation. Among the 397 cases of allo-HSCT, 177 were matched sibling transplantation, 165 were partially matched related transplantation, and 55 were matched unrelated transplantation. According to the stem cell source, there were 333 cases of peripheral hematopoietic origin, 55 from peripheral blood combined with bone marrow transplantation, and nine involved cord blood transplantation.

\section{Conditioning Regimen and Prevention of Graft-versus-Host Disease (GVHD)}

Central vein catheterization was performed for all patients before transplantation conditioning. Modified busulfan/ cyclophosphamide $(\mathrm{Bu} / \mathrm{Cy})$ and total body irradiation/cyclophosphamide (TBI/Cy) conditioning regimens were used for patients with acute leukemia, myelodysplastic syndrome, and lymphoma. Meanwhile, cyclophosphamide + anti-thymocyte globulin (Cy-ATG) and FluCy-ATG pre-treatment regimens were used for severe aplastic anemia. The GVHD prevention program used cyclosporine combined with mycophenolate mofetil and methotrexate, of which 272 cases were also treated with ATG to prevent GVHD.

\section{Infection Prevention and Treatment Strategies}

All HSCT patients were admitted to the laminar flow purification ward after a medicated bath, and were given a sterile diet, and received oral, eye, nose, and perianal care. Take a 1:2000 chlorhexidine liquid medicinal bath for 20 minutes; routinely gargle with saline and cermetium chloride before and after three meals a day, add metronidazole solution if necessary; use $1 \%$ chloramphenicol, $0.5 \%$ Rifampicin eye drops alternate eye drops, 4 times/d; alternate nose drops with houttuynia cordata and streptomycin nasal drops, 4 times/d; rinse the perineum with warm water after each bowel movement, 3\% boric acid solution for a bath for 20 Minutes, mupirocin is applied to the perianal area. Itraconazole, berberine, and compound sulfamethoxazole were administered orally for intestinal disinfection two weeks before transplantation. If the body temperature of patients got to $\geq 38.00^{\circ} \mathrm{C}$ during transplantation or shivering occurred, $10 \mathrm{~mL}$ of blood from the peripheral vein was collected using standard. The blood was drawn twice in a row for separate cultivation of aerobic and anaerobic bacteria. For positive cases, broad-spectrum antibiotics were administered intravenously, and the treatment efficacy was evaluated 48 hours after the initial treatment. Treatment efficacy was empirically assessed based on blood culture results, WBC, C-reactive protein, and procalcitonin levels, after which ineffective treatment strategies were adjusted.

\section{Diagnostic Criteria}

Agranulocytosis refers to the absolute value of neutrophils $<0.5 \times 10^{9} / \mathrm{L},{ }^{14}$ while granulocyte reconstitution refers to 
neutrophils $\geq 0.5 \times 10^{9} / \mathrm{L}$ for three consecutive days after transplantation.

Fever is a single measurement of oral temperature $\geq 38.3^{\circ} \mathrm{C}$ (axillary temperature $\geq 38.0^{\circ} \mathrm{C}$ ) or $\geq 38.0^{\circ} \mathrm{C}$ (axillary temperature $\geq 37.7^{\circ} \mathrm{C}$ ) for more than 1 hour.

The pathogenic diagnosis of BSI was made after the isolation of pathogenic microorganisms from blood culture. If the same patient isolates the same bacteria, if the drug sensitivity is the same, it is 1 BSI. BSI-related mortality was defined as death occurring within 30 days after the diagnosis of BSI. Pre-engraftment BSI is defined as the infection that arises from the onset of the pre-treatment regimen to the time before granulocyte implantation.

\section{Bacterial Culture Identification and Drug Sensitivity Test}

VersaTREK automatic blood culture instrument (Thermo Fisher, USA), VITEK MS IVD 3.0 mass spectrometer identification instrument and VITEK2 Compact automatic microbial identification, and drug sensitivity analysis system for bacterial culture, identification, and drug sensitivity detection, spread through paper (K-B) method and E-Test were used in in vitro susceptibility tests and review of abnormal susceptibility results. The results were interpreted according to the standards issued by the United States Committee for Clinical and Laboratory Standardization (CLSI). ${ }^{15}$

\section{Statistical Methods}

The SPSS21.0 software was used for statistical analysis, and descriptive statistics were used to summarize clinical features. The univariate analysis used a chi-square test, while logistic regression was applied for multivariate analysis. A P-value of 0.05 was used as the level of significance; thus, $\mathrm{P}<0.05$ indicated statistically significant differences.

\section{Results}

\section{The Incidence of BSI and the Positive Rate of Blood Culture}

Among the 397 HSCT patients, 294 had agranulocytosis fever, out of which 52 were microbiologically confirmed as BSIs. Therefore, the incidence of BSI was $17.7 \%(52 /$ $294)$, accounting for $13.1 \%(52 / 397)$ of all transplant patients. The implantation time of neutrophils is 13 days $(11,15)$, and the time from agranulocytosis to BSI is 12 days $(7,30)$. For 294 patients, we did 607 blood cultures, among which 60 were positive $(9.9 \%$ positive blood culture rate). Out of the 294 patients, six had two or more pathogenic bacteria.

\section{Pathogen Distribution and Drug Resistance}

Sixty pathogens were detected in 52 patients, including 43 Gram-negative bacteria (71.67\%), 10 Gram-positive bacteria (16.67\%), and 7 fungi (11.67\%). We found that Gram-negative bacteria accounted for most BSIs, followed by Gram-positive bacteria, and fungal infections were the least. The numbers and proportions of different strains of pathogenic bacteria are shown in Figure 1. In terms of drug resistance, the extended-spectrum $\beta$-lactamase (ESBL) detection rates of $E$. coli and $K$. pneumoniae were $46.7 \% \quad(7 / 15)$ and $30 \%$ (3/10), respectively. Carbapenem-resistant Enterobacteriaceae (CRE) accounted for $17.9 \%(5 / 28)$. The recorded patterns for Gram-negative bacteria drug susceptibility are shown in Table 1. The two staphylococci detected in Gram-positive bacteria were all methicillin-resistant, and all the three enterococci were sensitive to vancomycin, teicoplanin, and linezolid. The detected fungi belong to the genus Candida, and the resistance rates to itraconazole and voriconazole were $57.1 \%$ and $28.6 \%$, respectively.

\section{Treatment Status and Outcome}

Out of the 52 BSI patients, 33 improved after treatment, while 19 died after treatment failed (36.5\%). Among the 19, 13 had Gram-negative bacteria infection, three were Candida infections, while another three were mixed Gramnegative and Gram-positive bacterial infection. Six of the seven patients who were resistant to carbapenems died.

\section{Risk Factors for BSI}

We divided the 294 patients with agranular fever into two groups: BSI-free (242) and BSI (52). Univariate and multivariate analyses were applied for the study of BSI risk factors, including patients' age, gender, disease type, stem cell source, pre-treatment application of ATG, combined diarrhea, oral ulcers, and presence of granules. Univariate analysis results demonstrated that the occurrence of BSI was correlated to the transplantation method, pre-treatment application of ATG, agranulocytosis time ( $\geq 21$ days), and stem cell source (Table 2). Meanwhile, multivariate analysis showed that pre-treatment application of ATG, 


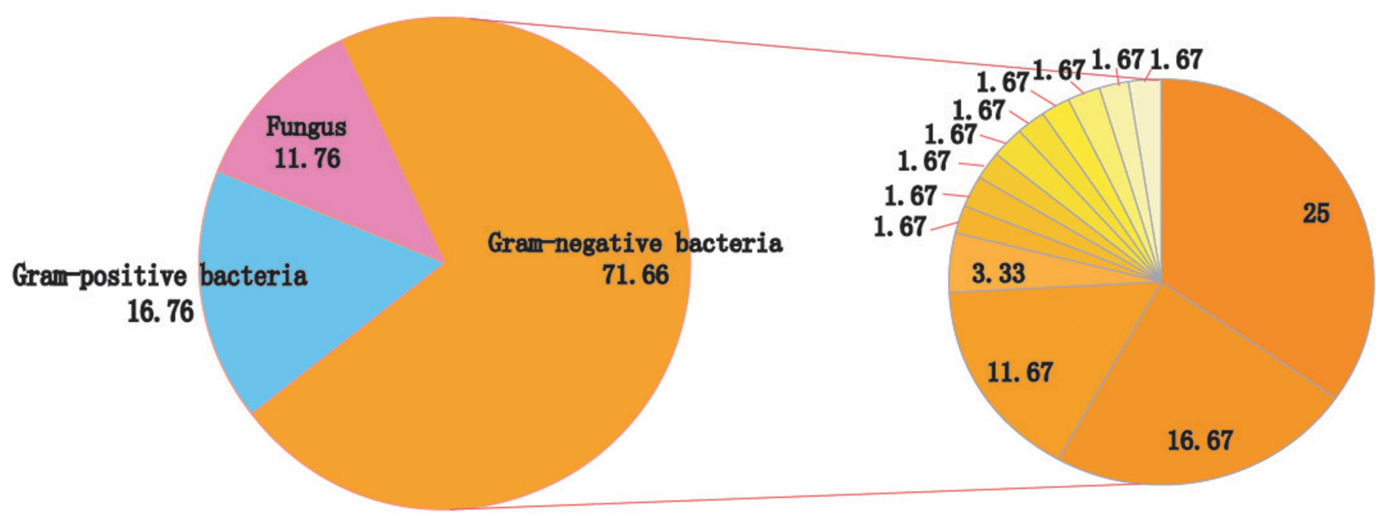

Figure I Distribution of 60 isolated pathogenic bacteria pathogen.

agranulocytosis time ( $\geq 21$ days), and stem cell source were risk factors for BSI (Table 3).

\section{Discussion}

Allo-HSCT patients undergo prolonged agranulocytosis and develop an impaired mucosal barrier. Besides, the long-term use of immunosuppressive agents increases the incidence of bloodstream infections. ${ }^{4-7}$ In the present study, the incidence of bloodstream infections was $13.1 \%$ in all patients, and $17.7 \%$ in patients with febrile neutropenia. A previous study conducted in China reported that the incidence of bloodstream infections in patients with febrile neutropenia was $17.0 \%{ }^{16}$ Thus, our findings are consistent with earlier results of other studies. The mortality rate of allo-HSCT bloodstream infections in our center was $36.5 \%$, which is higher than the $26.9 \%$ reported by Mikulska et $\mathrm{al}^{17}$ and the $31.1 \%$ reported by Stoma et al. ${ }^{18}$ In addition, studies by Stoma et al also found that the application of fluoroquinolones can reduce the incidence of bloodstream infections by affecting the colonization of intestinal bacteria, while insufficient empirical antibacterial treatment is associated with increased

Table I Resistance Rate of Major Gram-Negative Bacteria to Common Antibacterial Drugs

\begin{tabular}{|c|c|c|c|c|c|c|}
\hline \multirow[t]{2}{*}{ Antibacterial Drugs } & \multicolumn{2}{|c|}{ Escherichia coli } & \multicolumn{2}{|c|}{ Klebsiella pneumoniae } & \multicolumn{2}{|c|}{ Pseudomonas aeruginosa } \\
\hline & Number & Rate(\%) & Number & Rate(\%) & Number & Rate (\%) \\
\hline Piperacillin/Tazobactam & 5 & 33.3 & 5 & 50.0 & 4 & 57.1 \\
\hline Cefazolin & 14 & 93.3 & 6 & 60.0 & 5 & 83.3 \\
\hline Cefotetan & 5 & 35.7 & 3 & 33.3 & 6 & 100.0 \\
\hline Ceftazidime & 8 & 57.1 & 4 & 44.4 & 4 & 57.1 \\
\hline Ceftriaxone & 12 & 80.0 & 5 & 50.0 & 5 & 83.3 \\
\hline Cefepime & 7 & 46.7 & 4 & 40.0 & 4 & 57.1 \\
\hline Imipenem & 1 & 6.7 & 3 & 30.0 & 2 & 28.6 \\
\hline Amikacin & 2 & 13.3 & I & 10.0 & 1 & 14.3 \\
\hline Gentamicin & 10 & 66.7 & 2 & 20.0 & 5 & 71.4 \\
\hline Ciprofloxacin & 12 & 80 & 4 & 40.0 & 1 & 14.3 \\
\hline Levofloxacin & 11 & 73.3 & 4 & 40.0 & 1 & 14.3 \\
\hline Compound trimethoprim & 13 & 86.7 & 6 & 60.0 & 5 & 83.3 \\
\hline Tigecycline & 0 & 0 & 0 & 0 & - & - \\
\hline Cefoperazone/Sulbactam & 3 & 20.0 & - & - & - & - \\
\hline Meropenem & - & - & - & - & 2 & 28.6 \\
\hline
\end{tabular}


Table 2 Univariate Analysis of Risk Factors for BSI

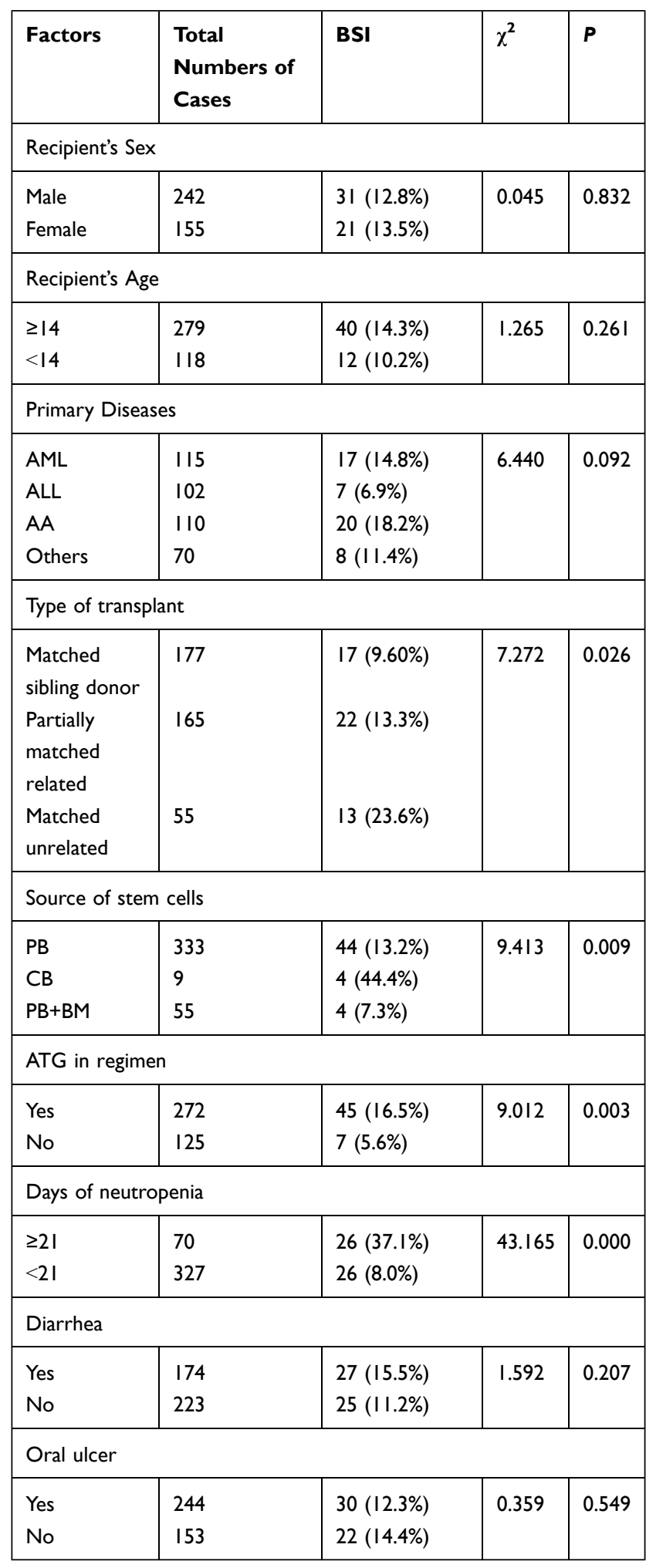

mortality. ${ }^{18,19}$ This disparity suggests that we should pay attention to the prevention and treatment of bloodstream infections in transplant patients and formulate anti- infection strategies based on the distribution of pathogens and drug resistance patterns to improve transplantation and survival rates.

This study detected 60 pathogens in BSIs, of which gram-negative bacteria $(71.67 \%)$ were the main ones, followed by gram-positive bacteria (16.67\%), and fungi were the least (11.67\%) (Figure 1). Gram-negative bacteria were mainly of the Enterobacteriaceae family, particularly E. coli and $K$. pneumoniae. The non-fermenting bacteria P. aeruginosa was also detected. A 25-year study in Spain showed that BSIs after HSCT were mainly caused by grampositive bacteria, with a downward trend in positive bacteria and an increasing trend in gram-negative bacteria. ${ }^{20}$ Blennow et al also reported similar conclusions. ${ }^{21}$ However, many transplant centers in China have reported that BSIs after HSCT are mainly caused by gram-negative bacteria, followed by gram-positive bacteria, while fungi make up the least proportion. Thus, the epidemiology of BSIs in our center conforms to the distribution pattern reported in other centers in China. ${ }^{22,23}$

In this study, the common Enterobacteriaceae (E. coli and $K$. pneumoniae) had ESBL detection rates of $46.7 \%$ and $30 \%$, respectively, and carbapenem resistance rates of the two bacteria were $6.7 \%$ and $30 \%$, respectively (Table $1)$. Thus, we found that E. coli is highly sensitive to carbapenem drugs, suggesting that these drugs can be used for empiric antibacterial treatment. The ESBL positivity rate and carbapenem resistance rate of K. pneumoniae were both $30 \%$ (Table 1), indicating that its clinical treatment can be a combination of tigecycline, polymyxin, and other drugs. Notably, research shows that combination therapy with antibacterial medications such as cyclin and polymyxin can reduce the mortality of patients. $^{24,25}$ In the present study, the resistance rate of

Table 3 Multivariate Analysis of Risk Factors for BSI

\begin{tabular}{|c|c|c|c|c|}
\hline Risk Factors & Wald & P-value & $\begin{array}{l}\text { Exp } \\
\text { (B) }\end{array}$ & $95 \% \mathrm{Cl}$ \\
\hline Type of transplant & 1.265 & 0.531 & - & - \\
\hline $\begin{array}{l}\text { Partially matched } \\
\text { related }\end{array}$ & 0.436 & 0.509 & 0.756 & $0.330-1.732$ \\
\hline Matched unrelated & 0.172 & 0.678 & 1.250 & $0.436-3.578$ \\
\hline Source of stem cells & 8.209 & 0.016 & - & - \\
\hline PB & 4.011 & 0.045 & 3.197 & $1.025-9.969$ \\
\hline $\mathrm{CB}$ & 7.875 & 0.005 & 24.784 & $2.633-233.279$ \\
\hline ATG in regimen & 4.832 & 0.028 & 3.401 & $1.142-10.132$ \\
\hline $\begin{array}{l}\text { Days of neutropenia } \\
\geq 21\end{array}$ & 28.265 & 0.000 & 6.407 & $3.231-12.707$ \\
\hline
\end{tabular}


P. aeruginosa to carbapenems was $28.6 \%$, while its resistance rate to both aminoglycosides and quinolones was $14.3 \%$ (Table 1). Thus, a combination of carbapenems, aminoglycosides, and quinolones can be used for clinical treatment. Multi-center research in China reported carbapenem resistance rates of $3.6 \%$ and $18.9 \%$ for $E$. coli and $K$. pneumoniae, respectively. ${ }^{26}$ Similarly, this study revealed high resistance of $E$. coli and $K$. pneumoniae to carbapenem. The high rate of mycene resistance could be attributed to the repeated use of broad-spectrum antibiotics in transplant patients and the continuous increase in multidrug-resistant bacteria in recent years. ${ }^{27}$ In response to the rise in multi-drug-resistant bacteria, our center uses perianal swabs to regularly screen intestine colonizing bacteria in transplant patients. As such, pathogenic bacteria are identified early, and treatment strategies are adjusted based on drug sensitivity results. The sensitivity of Grampositive bacteria to the glycopeptides vancomycin, linezolid, and teicoplanin was $100.0 \%$, suggesting that Grampositive bacteria BSIs can be completely treated in clinical practice. Thus, glycopeptide or azole drugs can be the first choice for the treatment of Gram-positive bacteria BSIs.

All the seven fungi in this study were Candida, and Candida tropicalis was the predominant species. The resistance rates to itraconazole and voriconazole were $57.1 \%$ and $28.6 \%$, respectively. The mortality rate of candidiasis was high, which significantly threatened the survival of transplant patients. According to previous studies, caspofungin should form the first choice fungal treatment after allo-HSCT in clinical practice, combined with antifungal treatment if necessary. ${ }^{28,29}$

The single-factor and multi-factor analysis results showed that pre-treatment application of ATG, agranulocytosis time ( $\geq 21$ days), and stem cell source were risk factors for BSI. The removal of T-lymphocytes from the body of ATG-pretreated patients significantly delays immune reconstitution, ${ }^{30}$ and the continued lack of granulocytes causes immunodeficiency in transplant patients, thus increasing the risk to BSIs. Peripheral blood combined with bone marrow transplantation, hematopoietic implantation is relatively fast, which may be the reason for the lower incidence of BSIs in this group of patients, relative to peripheral blood and cord blood transplantation. ${ }^{31-33}$

\section{Conclusions}

The results of this study show that BSI is a common complication of allo-HSCT patients with agranulocytosis. Gram-negative bacteria were the most prevalent pathogen in BSIs, and drug resistance to carbapenem drugs was relatively high. The use of ATG in pre-treatment, agranulocytosis time ( $\geq 21$ days), and stem cell source are risk factors for BSI. The high mortality rate of BSI substantially affects the prognosis of transplant patients, and attention should be paid on the distribution of pathogenic bacteria and drug resistance in the bloodstream of transplant patients. Besides, the treatment plan should be adjusted based on the specific bacteria and drug resistance patterns.

\section{Ethical Statement}

The patient consent was waived, since the research involves no more than minimal risk to the subjects because the review of subjects' medical records is for limited information. The information is not sensitive in nature, and the data are derived from clinically indicated procedures. The precautions taken to limit the record review to specified data and the coding of the data further minimize the primary risk, which is a breach of confidentiality. This study has been approved by the ethics review committee of the research project of the First Affiliated Hospital of Zhengzhou University, and has obtained relevant certificates.

\section{Author Contributions}

All authors made a significant contribution to the work reported, whether that is in the conception, study design, execution, acquisition of data, analysis and interpretation, or in all these areas; took part in drafting, revising or critically reviewing the article; gave final approval of the version to be published; have agreed on the journal to which the article has been submitted; and agree to be accountable for all aspects of the work. This study complies with the Declaration of Helsinki.

\section{Funding}

This project was supported by the Key Scientific Research Project Plan of Higher Education Institutions in Henan Province (18A320040).

\section{Disclosure}

The authors report no conflicts of interest in this work.

\section{References}

1. Barriga F, Ramirez P, Wietstruck A, Rojas N. Hematopoietic stem cell transplantation: clinical use and perspectives. Biol Res. 2012;45 (3):307-316. doi:10.4067/S0716-97602012000300012 
2. Passweg JR, Baldomero H, Bader P, et al. Use of haploidentical stem cell transplantation continues to increase: the 2015 European Society for Blood and Marrow Transplant activity survey report. Bone Marrow Transplant. 2017;52(6):811-817. doi:10.1038/bmt.2017.34

3. Gudiol C, Garcia-Vidal C, Arnan M, et al. Etiology, clinical features and outcomes of pre-engraftment and post-engraftment bloodstream infection in hematopoietic SCT recipients. Bone Marrow Transplant. 2014;49(6):824-830

4. Kikuchi M, Akahoshi Y, Nakano H, et al. Risk factors for pre- and post-engraftment bloodstream infections after allogeneic hematopoietic stem cell transplantation. Transpl Infect Dis. 2015;17(1):56-65.

5. Mori Y, Yoshimoto G, Nishida R, et al. Gastrointestinal Graft-versusHost Disease Is a Risk Factor for Postengraftment Bloodstream Infection in Allogeneic Hematopoietic Stem Cell Transplant Recipients. Biol Blood Marrow Transplant. 2018;24(11):2302-2309.

6. Mikulska M, Raiola AM, Galaverna F, et al. Pre-Engraftment Bloodstream Infections after Allogeneic Hematopoietic Cell Transplantation: impact of $\mathrm{T}$ Cell-Replete Transplantation from a Haploidentical Donor. Biol Blood Marrow Transplant. 2018;24 (1):109-118.

7. Weisser M, Theilacker C, Tschudin Sutter S, et al. Secular trends of bloodstream infections during neutropenia in 15181 haematopoietic stem cell transplants: 13-year results from a European multicentre surveillance study (ONKO-KISS). Clin Microbiol Infect. 2017;23 (11):854-859.

8. Poutsiaka DD, Munson D, Price LL, Chan GW, Snydman DR. Blood stream infection (BSI) and acute GVHD after hematopoietic SCT (HSCT) are associated. Bone Marrow Transplant. 2011;46 (2):300-307.

9. Youssef A, Hafez H, Madney Y, et al. Incidence, risk factors, and outcome of blood stream infections during the first 100 days post-pediatric allogeneic and autologous hematopoietic stem cell transplantations. Pediatr Transplant. 2020;24(1):e13610.

10. Wang CH, Chang FY, Chao TY, et al. Characteristics comparisons of bacteremia in allogeneic and autologous hematopoietic stem cell-transplant recipients with levofloxacin prophylaxis and influence on resistant bacteria emergence. J Microbiol Immunol Infect. 2018;51 (1):123-131. doi:10.1016/j.jmii.2016.02.003

11. Forcina A, Lorentino F, Marasco V, et al. Clinical Impact of Pretransplant Multidrug-Resistant Gram-Negative Colonization in Autologous and Allogeneic Hematopoietic Stem Cell Transplantation. Biol Blood Marrow Transplant. 2018;24 (7):1476-1482. doi:10.1016/j.bbmt.2018.02.021

12. Averbuch D, Tridello G, Hoek J, et al. Antimicrobial Resistance in Gram-Negative Rods Causing Bacteremia in Hematopoietic Stem Cell Transplant Recipients: intercontinental Prospective Study of the Infectious Diseases Working Party of the European Bone Marrow Transplantation Group. Clin Infect Dis. 2017;65 (11):1819-1828. doi:10.1093/cid/cix646

13. Girmenia C, Rossolini GM, Piciocchi A, et al. Infections by carbapenem-resistant Klebsiella pneumoniae in SCT recipients: a nationwide retrospective survey from Italy. Bone Marrow Transplant. 2015;50(2):282-288. doi:10.1038/bmt.2014.231

14. Freifeld AG, Bow EJ, Sepkowitz KA, et al. Clinical practice guideline for the use of antimicrobial agents in neutropenic patients with cancer: 2010 update by the infectious diseases society of america. Clin Infect Dis. 2011;52(4): e56-93.

15. Arendrup MC, Prakash A, Meletiadis J, Sharma C, Chowdhary A. Comparison of EUCAST and CLSI Reference Microdilution MICs of Eight Antifungal Compounds for Candida auris and Associated Tentative Epidemiological Cutoff Values. Antimicrob Agents Chemother. 2017;61(6):6. doi:10.1128/AAC.00485-17

16. Han TT, Huang XJ, Liu KY, et al. [Blood stream infections during agranulocytosis period after hematopoietic stem cell transplantation in one single center]. Zhonghua Nei Ke Za Zhi. 2011;50(8):654-658.
17. Mikulska M, Del Bono V, Bruzzi P, et al. Mortality after bloodstream infections in allogeneic haematopoietic stem cell transplant (HSCT) recipients. Infection. 2012;40(3):271-278. doi:10.1007/s15010-0110229-y

18. Stoma I, Karpov I, Milanovich N, Uss A, Iskrov I. Risk factors for mortality in patients with bloodstream infections during the pre-engraftment period after hematopoietic stem cell transplantation. Blood Res. 2016;51(2):102-106. doi:10.5045/br.2016.51.2.102

19. Stoma I, Littmann ER, Peled JU, et al. Compositional flux within the intestinal microbiota and risk for bloodstream infection with gram-negative bacteria. Clin Infect Dis. 2020. doi:10.1093/cid/ciaa068

20. Puerta-Alcalde P, Cardozo C, Marco F, et al. Changing epidemiology of bloodstream infection in a 25 -years hematopoietic stem cell transplant program: current challenges and pitfalls on empiric antibiotic treatment impacting outcomes. Bone Marrow Transplant. 2020;55 (3):603-612. doi:10.1038/s41409-019-0701-3

21. Blennow O, Ljungman P, Sparrelid E, Mattsson J, Remberger M. Incidence, risk factors, and outcome of bloodstream infections during the pre-engraftment phase in 521 allogeneic hematopoietic stem cell transplantations. Transpl Infect Dis. 2014;16(1):106-114. doi:10.1111/tid.12175

22. Liu C-Y, Lai Y-C, Huang L-J, et al. Impact of bloodstream infections on outcome and the influence of prophylactic oral antibiotic regimens in allogeneic hematopoietic SCT recipients. Bone Marrow Transplantation. 2020;55(3):1231-1239. doi:10.1038/bmt.2010.286

23. Wang L, Wang Y, Fan X, Tang W, Hu J. Prevalence of Resistant Gram-Negative Bacilli in Bloodstream Infection in Febrile Neutropenia Patients Undergoing Hematopoietic Stem Cell Transplantation: A Single Center Retrospective Cohort Study. Medicine. 2014;16(1):e1931. doi:10.1097/MD.0000000000001931

24. Tumbarello M, Viale P, Viscoli C, et al. Predictors of mortality in bloodstream infections caused by Klebsiella pneumoniae carbapenemase-producing K. pneumoniae: importance of combination therapy. Clin Infect Dis. 2012;55(7):943-950. doi:10.1093/cid/ cis588

25. Qureshi ZA, Paterson DL, Potoski BA, et al. Treatment outcome of bacteremia due to KPC-producing Klebsiella pneumoniae: superiority of combination antimicrobial regimens. Antimicrob Agents Chemother. 2012;56(4):2108-2113.

26. Zhang R, Liu L, Zhou H, et al. Nationwide Surveillance of Clinical Carbapenem-resistant Enterobacteriaceae (CRE) Strains in China. EBioMedicine. 2017;19:98-106.

27. Taur Y, Xavier JB, Lipuma L, et al. Intestinal domination and the risk of bacteremia in patients undergoing allogeneic hematopoietic stem cell transplantation. Clin Infect Dis. 2012;55(7):905-914.

28. Mousset S, Buchheidt D, Heinz W, et al. Treatment of invasive fungal infections in cancer patients-updated recommendations of the Infectious Diseases Working Party (AGIHO) of the German Society of Hematology and Oncology (DGHO). Ann Hematol. 2014;93 (1): 13-32.

29. de Naurois J, Novitzky-Basso I, Gill MJ, et al. Management of febrile neutropenia: ESMO Clinical Practice Guidelines. Ann Oncol. 2010;21(Suppl 5):v252-256.

30. Mackall C, Fry T, Gress R, et al. Background to hematopoietic cell transplantation, including post transplant immune recovery. Bone Marrow Transplant. 2009;44(8):457-462.

31. Ge J, Yang T, Zhang L, et al. The incidence, risk factors and outcomes of early bloodstream infection in patients with malignant hematologic disease after unrelated cord blood transplantation: a retrospective study. BMC Infect Dis. 2018;18(1):654.

32. Laughlin MJ, Eapen M, Rubinstein P, et al. Outcomes after transplantation of cord blood or bone marrow from unrelated donors in adults with leukemia. $N$ Engl J Med. 2004;351(22):2265-2275.

33. Rocha V, Labopin M, Sanz G, et al. Transplants of umbilical-cord blood or bone marrow from unrelated donors in adults with acute leukemia. $N$ Engl J Med. 2004;351(22):2276-2285. 


\section{Publish your work in this journal}

Infection and Drug Resistance is an international, peer-reviewed openaccess journal that focuses on the optimal treatment of infection (bacterial, fungal and viral) and the development and institution of preventive strategies to minimize the development and spread of resistance. The journal is specifically concerned with the epidemiology of

Submit your manuscript here: https://www.dovepress.com/infection-and-drug-resistance-journal antibiotic resistance and the mechanisms of resistance development and diffusion in both hospitals and the community. The manuscript management system is completely online and includes a very quick and fair peerreview system, which is all easy to use. Visit http://www.dovepress.com/ testimonials.php to read real quotes from published authors. 